\title{
Prevalencia de la hipoventilación relacionada con el sueño según diferentes criterios diagnósticos
}

\author{
Prevalence of sleep-related hypoventilation \\ according to different diagnostic criteria
}

\author{
Germán Díaz Santos, MD¹ Héctor Daniel Domínguez, Tec. ${ }^{2}$ Selene Guerrero Zúñiga, MD³
}

\section{Resumen}

INTRODUCCIÓN: la hipoventilación alveolar es una alteración que conduce a la retención de dióxido de carbono (CO2) y alteración en la presión arterial de $\mathrm{CO} 2(\mathrm{PaCO} 2)$ por múltiples causas (síndromes de hipoventilación). El diagnóstico es con gasometría arterial diurna en reposo con posible uso de subrogados, como la medición de dióxido de carbono transcutáneo $(\mathrm{TcCO} 2)$.

Todavía no está totalmente definida la estrategia diagnóstica, por lo cual nuestro objetivo fue evaluar la prevalencia de la hipoventilación durante el sueño utilizando diferentes criterios diagnósticos.

MATERIALES Y MÉTODOS: estudio observacional, descriptivo, retrospectivo de corte transversal de pacientes que requirieron polisomnografía entre 2015 y 2016 con medición de TcCO2 en el Instituto Nacional de Enfermedades Respiratorias Ismael Cosio Villegas de la Ciudad de México. Se les aplicó 6 diferentes criterios de hipoventilación durante el sueño. Se establecieron 3 grupos (sin trastornos respiratorios durante el sueño, pacientes con síndrome de hipoventilación-obesidad y pacientes con hipoventilación relacionada con alteración médica-neuromuscular). Se excluyeron pacientes que no tuvieran diagnóstico establecido. Se utilizó el dispositivo SenTec Digital Monitoring System para la medición del TcCO2, con el que se obtuvo el CO2 basal, $\mathrm{CO} 2$ promedio, $\mathrm{CO} 2$ máximo y tiempo de $\mathrm{CO} 2$. Se utilizó el programa SPSS v23. La descripción se realizó con media, mediana, desviación estándar y porcentajes según el tipo de variable. Se realizaron pruebas de concordancia entre los diferentes criterios aplicados para cada distribución. Se aceptó la significancia estadística cuando $\mathrm{p}<0,05$.

RESULTADOS: una muestra total de 143 pacientes, 51 sin trastornos respiratorios durante el sueño, 63 con síndrome de hipoventilación-obesidad y 29 con hipoventilación relacionada con alteraciones médicas (neuromusculares). Predominó el sexo masculino y eran adultos jóvenes. Un TcCO2 $>38 \mathrm{~mm} \mathrm{Hg}$ se diagnosticó al $68 \%$ de los pacientes con síndrome de hipoventilación-obesidad y el $69 \%$ de los pacientes con hipoventilación alveolar por enfermedades neuromusculares (Kappa de Cohen $=0,40$ ). Un $\mathrm{TcCO}_{2}>49 \mathrm{~mm} \mathrm{Hg}$ durante la noche diagnosticó al $82 \%$ de los pacientes con síndrome de hipoventilación-obesidad y el 58 $\%$ de los pacientes con hipoventilación alveolar por enfermedades neuromusculares (Kappa de Cohen $=0,63)$.

CONCLUSIÓN: en la población estudiada, un pico de TcCO2 $\geq 49 \mathrm{~mm} \mathrm{Hg}$ tiene un buen grado de acuerdo con el diagnóstico actual del paciente.

Palabras clave: síndromes de hipoventilación, hipoventilación alveolar, dióxido de carbono transcutáneo, hipoventilación durante el sueño.

\footnotetext{
'Internista, Neumólogo, Epidemiólogo, Especialista en Trastornos Respiratorios Durante el Dormir. Hospital Clínica San Rafael. Bogotá D. C., Colombia.

${ }^{2}$ Técnico en Sueño, Instituto Nacional de Enfermedades Respiratorias (INER), Coordinador del Laboratorio de la Clínica de trastornos del Sueño, Universidad Nacional Autónoma de México (UNAM). Ciudad de México.

${ }^{3}$ Neumóloga, Fisiología Pulmonar, Especialista en Trastornos Respiratorios Durante el Dormir. Instituto Nacional de Enfermedades Respiratorias (INER), Clínica de trastornos del Sueño. Ciudad de México.
} 


\section{Abstract}

INTRODUCTION: Alveolar hypoventilation is an alteration that leads to retention of carbon dioxide $\left(\mathrm{CO}_{2}\right)$ and alteration in blood pressure of $\mathrm{CO}_{2}\left(\mathrm{PaCO}_{2}\right)$ due to multiple causes (hypoventilation syndromes). The diagnosis is with resting daytime blood gasometry with possible use of other tests such as the measurement of transcutaneous carbon dioxide $\left(\mathrm{TcCO}_{2}\right)$.

The diagnostic strategy is not yet fully defined, so our objective was to evaluate the prevalence of hypoventilation during sleep using different diagnostic criteria.

MATERIALS AND METHODS: Observational, descriptive, retrospective, cross-sectional study of patients who required polysomnography between 2015 and 2016 with measurement of $\mathrm{TcCO}_{2}$ at the National Institute of Respiratory Diseases Ismael Cosio Villegas of Mexico City. Six different criteria of hypoventilation were applied during sleep. Three groups were established (without respiratory disorders during sleep, patients with hypoventilation-obesity syndrome and patients with hypoventilation related to medical-neuromuscular disorder). Patients who had no established diagnosis were excluded. The SenTec Digital Monitoring System was used to measure the $\mathrm{TcCO}_{2}$, obtaining baseline $\mathrm{CO}_{2}$, average $\mathrm{CO}_{2}$, maximum $\mathrm{CO}_{2}$ and $\mathrm{CO}_{2}$ time. The software SPSS v23 was used. The description was made with mean, median, standard deviation and percentages according to the type of variable. Concordance tests were performed between the different criteria applied for each distribution. Statistical significance was accepted when $\mathrm{p}<0.05$.

RESULTS: A total sample of 143 patients. 51 without respiratory disorders during sleep, 63 with hypoventilation-obesity syndrome and 29 with hypoventilation related to medical disorders (neuromuscular). The male gender predominated and they were young adults. $\mathrm{A} \mathrm{TcCO}_{2}>38 \mathrm{~mm} \mathrm{Hg}$ is diagnosed in $68 \%$ of patients with hypoventilation-obesity syndrome and $69 \%$ of patients with alveolar hypoventilation due to neuromuscular diseases (Cohen's $\mathrm{Kappa}=0.40$ ). A $\mathrm{TcCO}_{2}>49 \mathrm{~mm} \mathrm{Hg}$ at night diagnosed $82 \%$ of patients with hypoventilation-obesity syndrome and $58 \%$ of patients with alveolar hypoventilation due to neuromuscular diseases $($ Cohen's Kappa =0.63).

CONCLUSION: In the population studied, a peak of $\mathrm{TcCO}_{2} \geq 49 \mathrm{~mm} \mathrm{Hg}$ has a good degree according to the current diagnosis of the patient.

Keywords: Alveolar hypoventilation, hypoventilation syndromes, transcutaneous carbon dioxide, hypoventilation during sleep.

\section{Introducción}

La hipoventilación alveolar es una alteración en la ventilación que conduce a una retención de dióxido de carbono $\left(\mathrm{CO}_{2}\right)$ y alteración en la presión arterial de $\mathrm{CO}_{2}\left(\mathrm{PaCO}_{2}\right)(1)$. La pueden causar múltiples enfermedades como la enfermedad pulmonar obstructiva crónica (EPOC), síndrome de hipoventilación, obesidad, hipoventilación central, deformidades de la caja torácica y enfermedad neuromuscular, llevando a estados agudos o crónicos dependiendo el mecanismo, y se denominan síndromes de hipoventilación (2).

Una vez sospechada la hipoventilación se solicita una gasometría arterial en reposo diurna para establecer el diagnóstico, pero esta solo muestra el estado del intercambio gaseoso en dicho momento, además de la incomodidad y las complicaciones que causa la toma de la muestra. Otra alternativa nombrada en la literatura es la saturación de pulso nocturna; sin embargo, su sensibilidad es muy baja y en pacientes que requieren oxígeno suplementario no es fiable (3). Nuevas herramientas diagnósticas como la medición de dióxido de carbono transcutáneo $\left(\mathrm{TcCO}_{2}\right)$, que se ha aceptado como un subrogado de los gases arteriales, han permitido mejorar la oportunidad para realizar el diagnóstico de este tipo de enfermedades (4-6).

La ventilación mecánica no invasiva domiciliaria es el tratamiento de elección para estos pacientes y debe ser iniciada una vez confirmado el diagnóstico, ya que mejora la supervivencia a largo plazo $(7,8)$.

Existen 8 criterios (9-15) mencionados en la literatura con rentabilidad diagnóstica variable en diferentes tipos de pacientes. Se considera que todavía no está totalmente definida la estrategia diagnóstica para para hipoventilación relacionada con el sueño, por lo cual nuestro objetivo fue evaluar la prevalencia de la hipoventilación durante el sueño utilizando diferentes criterios diagnósticos. 


\section{Materiales y métodos}

Estudio observacional, descriptivo, retrospectivo de corte transversal de pacientes que requirieron polisomnografía entre 2015 y 2016 con medición de $\mathrm{TcCO}_{2}$ en el Instituto Nacional de Enfermedades Respiratorias "Ismael Cosio Villegas" de la Ciudad de México. Se les aplicó 6 diferentes criterios de hipoventilación durante el sueño.

\section{Selección de los pacientes}

Se tomaron todos los estudios de los pacientes durante 1 año, estableciéndose 3 grupos (sin trastornos respiratorios durante el sueño, pacientes con síndrome de hipoventilación-obesidad y pacientes con hipoventilación relacionada con alteración médica-neuromuscular).

Se excluyeron pacientes que no tuvieran diagnóstico establecido, pacientes del grupo de sanos con antecedentes de tabaquismo $\mathrm{u}$ otros problemas pulmonares, y pacientes del grupo de enfermos que cursaran con alguna alteración que aumentara el $\mathrm{CO}_{2}$.

El estudio se realizó de acuerdo con la Declaración de Helsinki y fue aprobado por el Comité de Ética de la institución.

\section{Estudio polisomnográfico}

Se les realizó a todos los pacientes una PSG estándar basal o de noche dividida, en una habitación parcialmente aislada y tranquila, con humedad y temperatura con baja variación. Generalmente, el estudio se inició a las 21 horas y finalizo a las 6 horas. El polígrafo utilizado fue GRASS de Dinámica Médica, S.A., que es distribuidor exclusivo en México para la compañía Astro-Med Grass-Telefactor, Inc., fabricante desde 1935 de electroencefalógrafos, polisomnógrafos (digitales y analógicos) que cuenta con sistemas de adquisición, digitalización y análisis de datos originados por señales analógicas (software PolyVIEW, GAMMA, TWIN). El polígrafo se utilizó con electrooculograma ( 2 canales), electroencefalograma (6 canales), electromiograma de los músculos submentonianos (2 canales), electromiograma del músculo tibial anterior de ambas piernas ( 2 canales), electrocardiograma y flujo de aire (con un termistor oronasal). Los movimientos torácicos y abdominales ( 2 canales) se registraron mediante pletismografía por inductancia y la determinación de la saturación arterial de oxihemoglobina $\left(\mathrm{SaO}_{2}\right)$, por medio de un sensor colocado en un dedo de la mano (principalmente el dedo índice). Para la calificación se utilizaron los criterios recomendados por la Academia Americana de Medicina del Sueño de 2012.

\section{Medición de $\mathrm{TcCO}_{2}$}

Para la medición del $\mathrm{TcCO}_{2}$ se utilizó el dispositivo SenTec Digital Monitoring System (Versión de software SMB SW-V08.00; MPB SW-V06.00). Este mide la tensión de $\mathrm{CO}_{2}$ en la superficie cutánea $\left(\mathrm{PcCO}_{2}\right)$ a $37{ }^{\circ} \mathrm{C}$ en pacientes de todas las edades. A partir de la $\mathrm{PcCO}_{2}$ medida es posible estimar por medio de un algoritmo la $\mathrm{PaCO}_{2}$.

Niveles de $\mathrm{TcCO}_{2}$ :

- $\mathrm{CO}_{2}$ basal: nivel promedio de $\mathrm{TcCO}_{2}$ despierto sentado en los primeros 2 minutos.

- $\mathrm{CO}_{2}$ promedio: nivel de $\mathrm{TcCO}_{2}$ marcado desde las luces apagadas hasta las luces prendidas.

- $\mathrm{CO}_{2}$ máximo: nivel de $\mathrm{TcCO}_{2}$ máximo alcanzado durante el sueño.

- Tiempo de $\mathrm{CO}_{2}$ : tiempo en minutos en que el paciente tiene un rango determinado de $\mathrm{CO}_{2}$.

\section{Criterios diagnósticos de hipoventilación}

Para definir la hipoventilación durante el sueño, se utilizaron los siguientes criterios diagnósticos:

1. Teniendo en cuenta que la presión parcial arterial de $\mathrm{CO}_{2}$ diurna $>45 \mathrm{~mm} \mathrm{Hg}\left(\mathrm{PaCO}_{2}>45 \mathrm{~mm} \mathrm{Hg}\right)$ es el criterio estandarizado más aceptado en la práctica clínica (9) y que el valor de un $\mathrm{CO}_{2}$ en los gases arteriales tiene buena correlación con el $\mathrm{TcCO}_{2}$, se tomó este mismo corte simulando este criterio.

2. Medición de $\mathrm{TcCO}_{2}$ superior a $55 \mathrm{~mm} \mathrm{Hg}\left(\mathrm{TcCO}_{2}\right.$ $>55 \mathrm{~mm} \mathrm{Hg}$ ) durante $\geq 10$ minutos (13).

3. Aumento del $\mathrm{TcCO}_{2}$ en $10 \mathrm{~mm} \mathrm{Hg}$ o más $\left(\mathrm{TcCO}_{2}\right.$ $\geq 10 \mathrm{~mm} \mathrm{Hg}$ ) comparado con el valor en supino despierto, y que sea superior a $50 \mathrm{~mm} \mathrm{Hg}$ durante $\geq 10$ minutos (14). 
4. Medición de $\mathrm{TcCO}_{2} \geq 49 \mathrm{~mm} \mathrm{Hg}$ durante cualquier momento de la noche $\left(\mathrm{TcCO}_{2} \geq 49 \mathrm{~mm} \mathrm{Hg}\right)(15)$.

5. Medición de $\mathrm{TcCO}_{2}$ promedio superior a $50 \mathrm{~mm} \mathrm{Hg}$ ( $\mathrm{TcCO}_{2}>50 \mathrm{~mm} \mathrm{Hg}$ ) (9).

6. Se tomó en cuenta que la $\mathrm{PaCO}_{2}$ diurna $\geq 38 \mathrm{~mm} \mathrm{Hg}$ $\left(\mathrm{PaCO}_{2} \geq 38 \mathrm{~mm} \mathrm{Hg}\right)$ en la práctica clínica normal en la Ciudad de México se considera hipercapnia, según estudios previos (16), y se consideró que el $\mathrm{TcCO}_{2}$ tiene una adecuada correlación con los gases.

El último criterio fue tomado de una publicación realizada en el 2000 para la Ciudad de México, refiriéndose a la gasometría normal a la altura de la ciudad. De acuerdo con estos criterios se realizó el análisis estadístico.

\section{Análisis estadístico}

Para el análisis estadístico se utilizó el programa SPSS v23 (IBM Ltd. v23.0 Statistics for Windows). E1 análisis descriptivo se realizó utilizando media, mediana y desviación estándar para las variables cuantitativas, y porcentajes para las cualitativas. Se realizaron pruebas de concordancia entre los diferentes criterios aplicados. Se aceptó la significancia estadística cuando $p<0,05$.

\section{Resultados}

Se reclutaron ciento cuarenta y tres pacientes $(n=$ 143) en total. Se distribuyeron en grupos: 51 sin trastornos respiratorios durante el sueño, 63 con síndrome de hipoventilación-obesidad y 29 con hipoventilación rela- cionada con alteraciones médicas (neuromusculares). Las características principales están descritas en la Tabla 1.

El sexo masculino fue predominante en los pacientes con enfermedad neuromuscular. Los pacientes sin trastorno respiratorio durante el sueño y los enfermos neuromusculares eran adultos jóvenes (Tabla 2).

La eficiencia de sueño fue baja en los pacientes enfermos. Se presentaron altos niveles de la etapa 1 y bajo porcentaje de etapa R. Los pacientes con síndrome de hipoventilación-obesidad tenían más hipoxia e hipercapnia.

El criterio diagnóstico número 6 detectó un gran porcentaje de personas sanas como enfermas. Todos los 6 criterios diagnósticos reportaron grandes porcentajes de síndrome de hipoventilación-obesidad como enfermos; sin embargo, el que más reportó fue el criterio diagnóstico número 5 , y el que menos porcentaje tuvo fue el número 4. El criterio diagnóstico número 5 fue el que menos reportó hipoventilación en los pacientes neuromusculares y el que reportó más fue el criterio diagnóstico número 4 (Figura 1, Tabla 3).

Se encontró que usando un punto de corte de $\mathrm{TcCO}_{2}$ $>38 \mathrm{~mm} \mathrm{Hg}$ se diagnostica al $68 \%$ de los pacientes con síndrome de hipoventilación-obesidad y al $69 \%$ de los pacientes con hipoventilación alveolar por enfermedades neuromusculares (Kappa de Cohen $=0,40$ ).

Un pico de $\mathrm{TcCO}_{2} \geq 49 \mathrm{~mm} \mathrm{Hg}$ durante la noche diagnosticó al $82 \%$ de los pacientes con síndrome de hipoventilación-obesidad y al $58 \%$ de los pacientes con hipoventilación alveolar por enfermedades neuromusculares (Kappa de Cohen $=0,63$ ).

Tabla 1. Características demográficas de los pacientes

\begin{tabular}{|lcccc|}
\hline Características & Total & Sin TRD & $\begin{array}{c}\text { Síndrome de } \\
\text { obesidad-hipoventilación }\end{array}$ & Neuromuscular \\
\hline N & 143 & 51 & 63 & 29 \\
Sexo masculino (\%) & 46,9 & 54,9 & 46 & 79,3 \\
Edad (años) & 39,9 & 24,2 & 56,4 & 29,4 \\
\hline
\end{tabular}

TRD: trastorno respiratorio del dormir. 
Tabla 2. Características demográficas y polisomnográficas de los pacientes

\begin{tabular}{|c|c|c|c|c|}
\hline & Total & Sin TRD & $\begin{array}{c}\text { Síndrome de } \\
\text { obesidad-hipoventilación }\end{array}$ & Neuromuscular \\
\hline \multicolumn{5}{|c|}{ Características demográficas } \\
\hline $\mathrm{N}$ & 143 & 51 & 63 & 29 \\
\hline Sexo masculino (\%) & 46,9 & 54,9 & 46 & 79,3 \\
\hline Edad (años) & 39,9 & 24,2 & 56,4 & 29,4 \\
\hline \multicolumn{5}{|c|}{ Características polisomnográficas } \\
\hline $\mathrm{N}$ & 143 & 51 & 63 & 29 \\
\hline Eficiencia (\%) & 78,7 & 85,3 & 71,7 & 79,1 \\
\hline Latencia a sueño (minutos) & 15,1 & 16,1 & 10,7 & 23,1 \\
\hline Etapa 1 (\%) & 19,4 & 10,3 & 29,1 & 14,2 \\
\hline Etapa $2(\%)$ & 50,8 & 48,8 & 51,2 & 51,3 \\
\hline Etapa $3(\%)$ & 18,3 & 22,4 & 12,7 & 22,5 \\
\hline Etapa R (\%) & 11,3 & 16,3 & 6,8 & 11,5 \\
\hline Saturación basal (\%) & 90,3 & 93 & 86,1 & 91,7 \\
\hline Saturación promedio (\%) & 86,7 & 92 & 80,5 & 87,8 \\
\hline $\mathrm{TcCO}_{2}$ basal $(\mathrm{mm} \mathrm{Hg})$ & 39,1 & 35,2 & 41,5 & 39,3 \\
\hline $\mathrm{TcCO}_{2}$ promedio $(\mathrm{mm} \mathrm{Hg})$ & 36,4 & 21,6 & 44,7 & 42,8 \\
\hline $\mathrm{TcCO}_{2}$ máximo $(\mathrm{mm} \mathrm{Hg})$ & 48,3 & 40,6 & 52,2 & 51,8 \\
\hline $\mathrm{IAH}$ (eventos/hora) & 35,6 & 1,6 & 67,3 & 26,3 \\
\hline
\end{tabular}

$\mathrm{IAH}$ : índice de apnea/hipopnea.

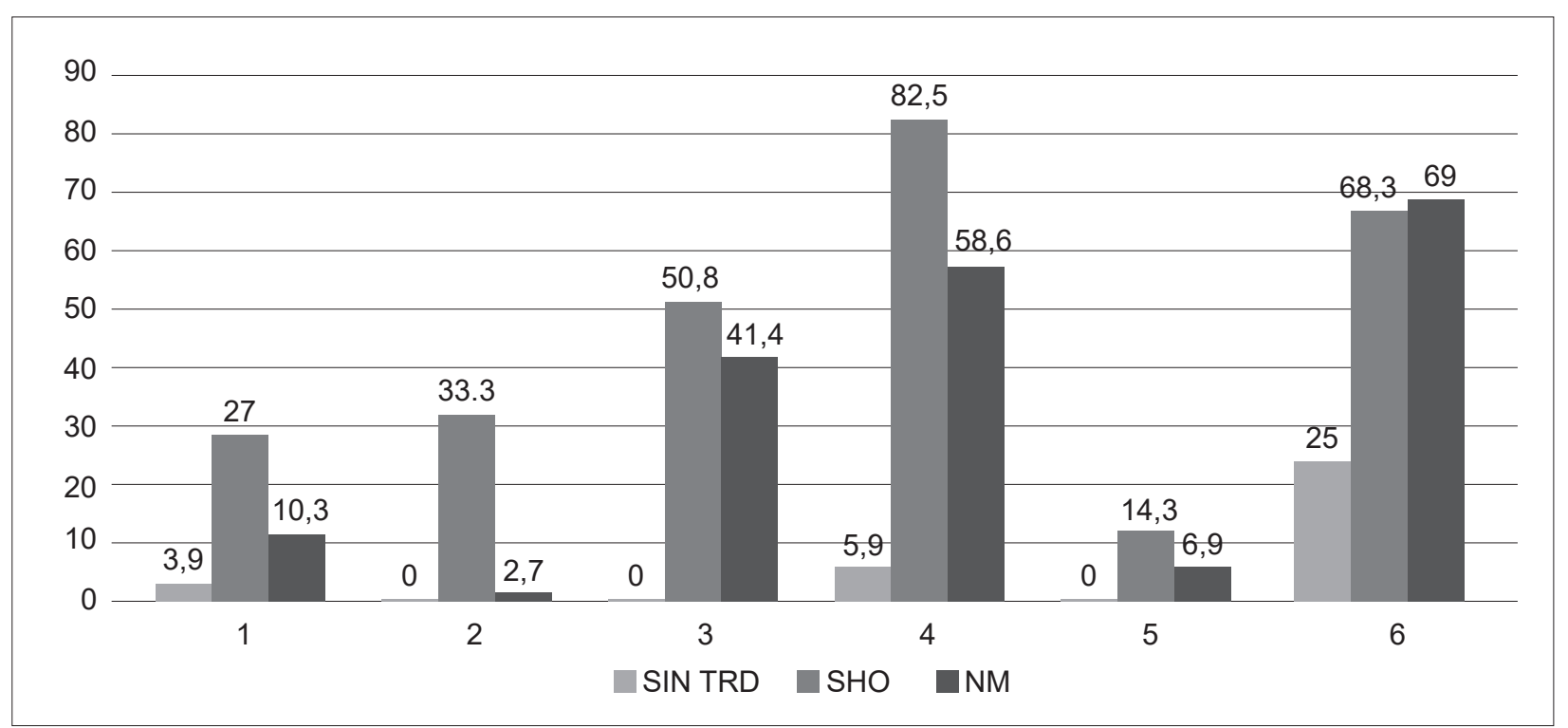

Figura 1. Porcentaje de diagnóstico con cada uno de los 6 criterios utilizados. Criterio diagnóstico 1: $\left(\mathrm{TcCO}_{2}>45 \mathrm{~mm}\right.$ $\mathrm{Hg}$ en la medición basal) (9), criterio diagnóstico 2: ( $\mathrm{TcCO}_{2}>55 \mathrm{~mm} \mathrm{Hg}$ ) durante $\geq 10$ minutos (13), criterio diagnóstico 3: $\left(\mathrm{TcCO}_{2} \geq 10 \mathrm{~mm} \mathrm{Hg}\right.$ comparado con el valor en supino despierto, y que sea superior a $50 \mathrm{~mm} \mathrm{Hg}$ durante $\geq 10$ minutos) (14), criterio diagnóstico 4: $\left(\mathrm{TcCO}_{2} \geq 49 \mathrm{~mm} \mathrm{Hg}\right)(15)$, criterio diagnóstico 5: ( $\left.\mathrm{TcCO}_{2}>50 \mathrm{~mm} \mathrm{Hg}\right)(9)$, criterio diagnóstico 6: ( $\mathrm{TcCO}_{2} \geq 38 \mathrm{~mm} \mathrm{Hg}$ en la medición basal) (16). NM: enfermedad neuromuscular; SHO: síndrome de hipoventilación-obesidad. 
Tabla 3. Correlación entre los diferentes criterios diagnósticos

\begin{tabular}{|lcc|}
\hline \multicolumn{1}{c}{ Criterio } & Kappa de Cohen & Valor de $\boldsymbol{p}$ \\
$\mathrm{TcCO}_{2}>45 \mathrm{~mm} \mathrm{Hg}$ & 0,136 & 0,005 \\
$\mathrm{TcCO}_{2}>55 \mathrm{~mm} \mathrm{Hg}$ por más de 10 minutos & 0,229 & 0,000 \\
$\mathrm{TcCO}_{2} \geq 10 \mathrm{~mm} \mathrm{Hg}$ que exceda $50 \mathrm{~mm} \mathrm{Hg}$ por más de $10 \mathrm{~min}$ & 0,395 & 0,000 \\
\hline Pico de $\mathrm{TcCO}_{2} \geq 49 \mathrm{~mm} \mathrm{Hg}$ & 0,636 & 0,000 \\
\hline Promedio $\mathrm{TcCO}_{2}>50 \mathrm{~mm} \mathrm{Hg}$ & 0,088 & 0,010 \\
$\mathrm{TcCO}_{2}>38 \mathrm{~mm} \mathrm{Hg}$ & 0,402 & 0,000 \\
\hline
\end{tabular}

\section{Discusión}

Los criterios que definen la hipoventilación durante el sueño son opiniones de expertos y no tienen una evidencia contundente; sin embargo, se requieren para el inicio de la ventilación mecánica no invasiva. Nuestro estudio intentó comparar las diferentes definiciones más utilizadas en la práctica clínica para el diagnóstico de hipoventilación durante el sueño, en pacientes de una clínica de sueño en la ciudad del México, teniendo en cuenta que está ubicada a 2250 metros por encima del nivel del mar y que la medición de $\mathrm{TcCO}_{2}$ es actualmente aceptada.

No se conoce exactamente el momento del inicio de la ventilación mecánica no invasiva, para elegir el mejor criterio para el inicio, porque la instauración temprana posiblemente disminuya todas las complicaciones (17).

Los principales criterios para el inicio de ventilación mecánica no invasiva son la hipercapnia diurna o la desaturación nocturna, los cuales se tomaron a su vez de las recomendaciones del grupo Medicare para el tratamiento con oxígeno en pacientes con EPOC (18).

Se ha reportado que hasta un tercio de los pacientes con normocapnia diurna presenta hipoventilación durante el sueño (19), pero esto mismo depende de qué criterio se tomó para definir la hipoventilación nocturna. En nuestro estudio, la prevalencia de la hipoventilación nocturna fue mayor utilizando un punto de corte de $\mathrm{TcCO}_{2} \geq 49 \mathrm{~mm} \mathrm{Hg}$.

No se consideró utilizar los criterios de desaturación, porque los pacientes que no presentan alteración del parénquima pulmonar en la curva de disociación de la hemoglobina no presentan grandes cambios en la saturación cuando el paciente está hipoventilando (20).

Los pacientes con insuficiencia respiratoria de origen restrictivo tienen como principal manifestación la hipoventilación, por lo que es importante identificar la hipoventilación durante el sueño, ya que el inicio de la ventilación mecánica no invasiva tiene una influencia sobre la morbimortalidad (21). Estudios metodológicamente débiles muestran que el uso de la presión positiva domiciliaria es el tratamiento ideal de la hipoventilación porque mejora la clínica del paciente, disminuye el riesgo de hospitalización y mortalidad, y mejora la calidad de vida (22). Sin embargo, la evidencia al momento no está tan clara ya que tienen estudios a favor y en contra. Un ensayo clínico aleatorizado controlado de pacientes con enfermedad neuromuscular sin hipoventilación diurna, pero con hipoventilación nocturna (15) mostró que el grupo que no recibió ventilación mecánica no invasiva presentó un deterioro en la calidad de vida independiente de la aparición de la hipoventilación diurna a 24 meses. Otro ensayo clínico aleatorizado controlado en pacientes con distrofia muscular de Duchenne, asintomáticos con función pulmonar comprometida, asignándolos a ventilación mecánica no invasiva encontró un aumento en la mortalidad en el grupo que recibió la terapia (este grupo tenía alteraciones cardíacas, no se tuvo en cuenta la adherencia al tratamiento y no se habían descartado trastornos respiratorios durante el sueño) (17).

evidenció la necesidad de iniciar ventilación mecánica no invasiva con un pico de $\mathrm{TcCO}_{2} \geq 49 \mathrm{~mm} \mathrm{Hg}$ durante el sueño a pesar de no tener hipoventilación 
diurna (7). Este valor fue el mismo que evidenció una adecuada correlación con el diagnóstico actual del paciente, por lo que consideramos que este valor sería una opción ideal para utilizar en la Ciudad de México.

Las limitaciones del estudio son por su naturaleza de diseño transversal retrospectivo, que solo permite describir el impacto de las diferentes definiciones probadas sobre la prevalencia de hipoventilación, sin la posibilidad de realizar otras evaluaciones. Otra limitación es la utilización de un subrogado del estándar de oro para el diagnóstico de la hipoventilación relacionada con el sueño, como lo es el $\mathrm{TcCO}_{2}$; pero existen trabajos que demuestran muy buena correlación de este con la prueba de oro (17), aunque pueden sobrestimar el valor de $\mathrm{CO}_{2}$ con el uso por períodos prolongados (23).

\section{Conclusión}

El uso de diferentes criterios diagnósticos cambia la prevalencia de la hipoventilación relacionada con el sueño. Sin embargo, para nuestros pacientes, un pico de $\mathrm{TcCO}_{2} \geq 49 \mathrm{~mm} \mathrm{Hg}$ tiene un buen grado de acuerdo con el diagnóstico actual del paciente.

\section{Referencias}

7. Piper AJ, Yee BJ. Hypoventilation syndromes. Compr Physiol. 2014;4(4):1639-76.

8. Fayyaz J, Lessnau KD, Hoo G, Byrd R, Hayes JA, Sharma $\mathrm{OP}$, et al. Hypoventilation syndromes. Medscape [internet] actualizado el 22 de septiembre de 2016 [acceso el 22 de enero de 2019]. Disponible en: http://emedicine.medscape.com/ article/304381-overview.

9. Fu ES, Downs JB, Schweiger JW, Miguel RV, Smith RA. Supplemental oxygen impairs detection of hypoventilation by pulse oximetry. Chest. 2004;126(5):1552-8.

10. Cuvelier A, Grigoriu B, Molano LC, Muir JF. Limitations of transcutaneous carbon dioxide measurements for assessing longterm mechanical ventilation. Chest. 2005;127(5):1744-8.

11. Janssens JP, Perrin E, Bennani I, de Muralt B, Titelion $\mathrm{V}$, Picaud C. Is continuous transcutaneous monitoring of PCO2 (TcPCO2) over $8 \mathrm{~h}$ reliable in adults? Respir. Med. 2001;95(5):331-5.

12. Storre JH, Steurer B, Kabitz HJ, Dreher M, Windisch W. Transcutaneous PCO2 monitoring during initiation of noninvasive ventilation. Chest. 2007;132(6):1810-6.

13. Annane D, Orlikowski D, Chevret S. Nocturnal mechanical ventilation for chronic hypoventilation in patients with neu- romuscular and chest wall disorders. Cochrane Database Syst Rev. 2014;(12):CD001941.

14. Bach JR, Gonçalves MR, Hon A, Ishikawa Y, De Vito EL, Prado F, et al. Changing trends in the management of endstage neuromuscular respiratory muscle failure: recommendations of an international consensus. Am J Phys Med Rehabil. 2013;92(3):267-77.

15. Simonds AK. Chronic hypoventilation and its management. Eur Respir Rev. 2013;22(129):325-32.

16. Hukins CA, Hillman DR. Daytime predictors of sleep hypoventilation in Duchenne muscular dystrophy. Am J Respir Crit Care Med. 2000;161(1):166-70.

17. Nardi J, Prigent H, Adala A, Bohic M, Lebargy F, Quera-Salva MA, et al. Nocturnal oximetry and transcuta-neous carbon dioxide in home-ventilated neuromuscular patients. Respir Care. 2012;57(9):1425-30.

18. Janssens JP, Borel JC, Pépin JL; SomnoNIV Group. Nocturnal monitoring of home non-invasive ventilation: the contribution of simple tools such as pulse oximetry, capnography, built-in ventilator software and autonomic markers of sleep fragmentation. Thorax. 2011;66(5):438-45.

19. Berry RB, Budhiraja R, Gottlieb DJ, Gozal D, Iber C, Kapur $\mathrm{VK}$, et al. Rules for scoring respiratory events in sleep: update of the 2007 AASM Manual for the Scoring of Sleep and Associated Events. Deliberations of the Sleep Apnea Definitions Task Force of the American Academy of Sleep Medicine. J Clin Sleep Med. 2012;8(5):597-619.

20. Aboussouan LS. Sleep-disordered Breathing in Neuromuscular Disease. Am J Respir Crit Care Med. 2015;191(9):979-89.

21. Ward S, Chatwin M, Heather S, Simonds AK. Randomised controlled trial of non-invasive ventilation (NIV) for nocturnal hypoventilation in neuromuscular and chest wall disease patients with daytime normocapnia. Thorax. 2005;60(12):1019-24.

22. Vázquez García JC, Pérez Padilla R. Valores gasométricos estimados para las principales poblaciones y sitios a mayor altitud en México. Rev Inst Nal Enf Resp Mex. 2000;13(1):6-13.

23. Raphael JC, Chevret S, Chastang C, Bouvet F. Randomised trial of preventive nasal ventilation in Duchenne muscular dystrophy. French Multicentre Cooperative Group on Home Mechanical Ventilation Assistance in Duchenne de Boulogne Muscular Dystrophy. Lancet 1994;343(8913):1600-4.

24. Storre JH, Magnet FS, Dreher M, Windisch W. Transcutaneous monitoring as a replacement for arterial $\mathrm{PCO}(2)$ monitoring during nocturnal non-invasive ventilation. Respir Med. 2011;105(1):143-50.

25. Gerdung CA, Adeleye A, Kirk VG. Noninvasive monitoring of $\mathrm{CO} 2$ during polysomnography: a review of the recent literature. Curr Opin Pulm Med. 2016;22(6):527-34.

26. Fletcher EC, Scott D, Qian W, Luckett RA, Miller CC, Goodnight-White S. Evolution of nocturnal oxyhemoglobin desaturation in patients with chronic obstructive pulmonary disease and a daytime $\mathrm{PaO} 2$ above $60 \mathrm{~mm} \mathrm{Hg}$. Am Rev Respir Dis. 1991;144(2):401-5. 
27. Eagle M, Baudouin SV, Chandler C, Giddings DR, Bullock R, Bushby K. Survival in Duchenne muscular dystrophy: improvements in life expectancy since 1967 and the impact of home nocturnal ventilation. Neuromuscul Disord. 2002;12(10):926-9.

28. Nickol AH, Hart N, Hopkinson NS, Moxham J, Simonds A, Polkey MI. Mechanisms of improvement of respiratory fai- lure in patients with restrictive thoracic disease treated with non-invasive ventilation. Thorax. 2005;60(9):754-60.

29. Berlowitz DJ, Spong J, O’Donoghue FJ, Pierce RJ, Brown DJ, Campbell DA, et al. Transcutaneous measurement of carbon dioxide tension during extended monitoring: evaluation of accuracy and stability, and an algorithm for correcting calibration drift. Respir Care. 2011;56(4):442-8. 